\section{Tetanus: The Old Enemy Resurfaces}

Hemanth Krishna Boppana ${ }^{1}$, Andrew Chan Chow ${ }^{2}$, Tarika Maharaj ${ }^{2}$, Sherry Sandy ${ }^{1}$, Daynish Maharaj ${ }^{1}$, Avidesh Panday $^{3}$, Surujpal Teelucksingh ${ }^{2}$

${ }^{1}$ Port-of-Spain General Hospital, Port-of-Spain, Trinidad

${ }^{2}$ Medical Associates Hospital, St. Joseph, Trinidad

${ }^{3}$ Eric Williams Medical Sciences Complex, Champ Fleurs, Trinidad

\section{Corresponding author}

Dr. Hemanth Krishna Boppana

Port-of-Spain General Hospital,

Port-of-Spain, Trinidad

E-mail: hemanth.boppana@hotmail.com

\section{DOI: $\underline{\text { doi.org/10.48107/CMJ.2021.04.004 }}$}

Copyright: This is an open-access article under the terms of the Creative Commons Attribution License which permits use, distribution, and reproduction in any medium, provided the original work is properly cited.

(C)2021 The Authors. Caribbean Medical Journal published by Trinidad \& Tobago Medical Association

\section{ABSTRACT}

The widespread use of the tetanus toxoid vaccine has been very effective in the prevention of tetanus. However, older patients who may have failed to receive or complete immunization schedules for this vaccine are prone to the significant risks of this life-threatening illness. We present two cases stemming from diabetic foot ulcers to remind clinicians of the presentation and treatment of this now rare disease and to also draw attention to the need to emphasise immunisation as a prevention strategy.

\section{CASE PRESENTATIONS}

\section{Case 1}

A 70-year-old Trinidadian male of African descent with a 10 -year history of diabetes on metformin and gliclazide presented with a one-day history of trismus and progressive left foot third digit gangrene for the past two months along with subjective fevers and progressive dysphagia for the last two days. He denied chest pain, dyspnoea, cough, fever or weight loss and reported healthy bladder and bowel function. The patient had a left great toe amputation eight months ago and his last documented immunisation was over 30 years ago. Vital signs were within normal limits with a respiratory rate of 16 breaths per minute and oxygen saturation on room air of $99 \%$. On physical examination, the head and neck showed risus sardonicus (Figure 1). His left foot third digit was gangrenous with a foul-smelling discharge. Of note, the patient developed a reflex spasm when a spatula was used to touch the oropharynx leading to the biting of the spatula which indicated a positive spatula test. Laboratory investigations showed a WBC of $11 \times 10^{9} / \mathrm{L}$ with mild leucocytosis and haemoglobin, platelets, blood urea nitrogen (BUN), creatinine and electrolytes within normal limits. C-reactive protein (CRP) was $1.8 \mathrm{mg} / \mathrm{L}$ (Normal < 1.0), with a HbA1C of $7.2 \%$ and normal creatine phosphokinase (CPK). X-rays of the left foot (anteroposterior and lateral) showed absence of soft tissue emphysema and osteopenia.

The patient was admitted to the High Dependency Unit (HDU) and a diagnosis of tetanus was made secondary to left diabetic foot gangrene. The patient was started on 
Figure 1 showing risus sardonicus

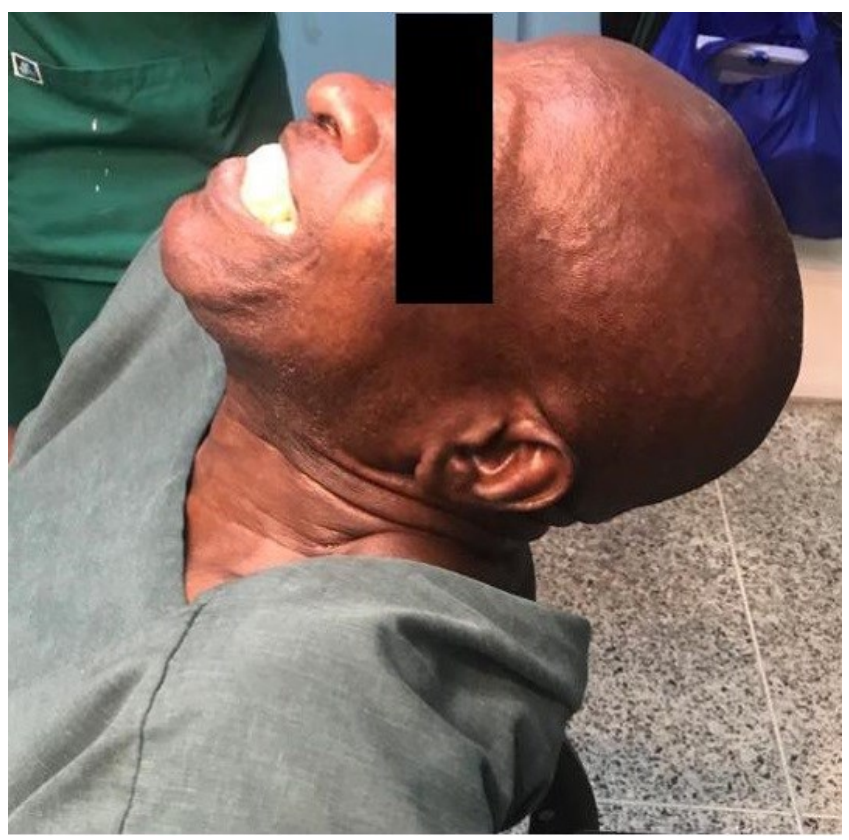

amoxicillin and metronidazole and aliquots of diazepam were administered every 4 to 6 hours to counter the trismus. Six thousand units of tetanus immune globulin were sourced and given intramuscularly after surgical debridement was carried out. There was no change in trismus 24 hours after admission. The patient developed opisthotonos and periods of apnea 48 hours after admission which progressed to respiratory distress from contraction of thoracic muscles and glottal/pharyngeal muscle contraction from tetanus toxin. An emergency tracheostomy was performed under general anaesthesia and the patient was placed on the ventilator and transferred to the Intensive Care Unit (ICU). The patient had a prolonged ICU course, complicated by pneumonia, where he was on mechanical ventilation for four weeks. He was gradually weaned off, then discharged and allowed home.

\section{Case 2}

A 68-year-old Trinidadian male of East Indian descent was referred from his general practitioner due to a progressively worsening left foot plantar aspect wound that occurred after a metal fragment punctured his skin one week prior (Figure 2). Past medical history was significant for diabetes mellitus for which he was noncompliant with his insulin regimen. He also had hypertension, stage 3 chronic kidney disease and coronary artery disease with a history of a prior

\section{Figure 2 showing the left foot plantar aspect wound}

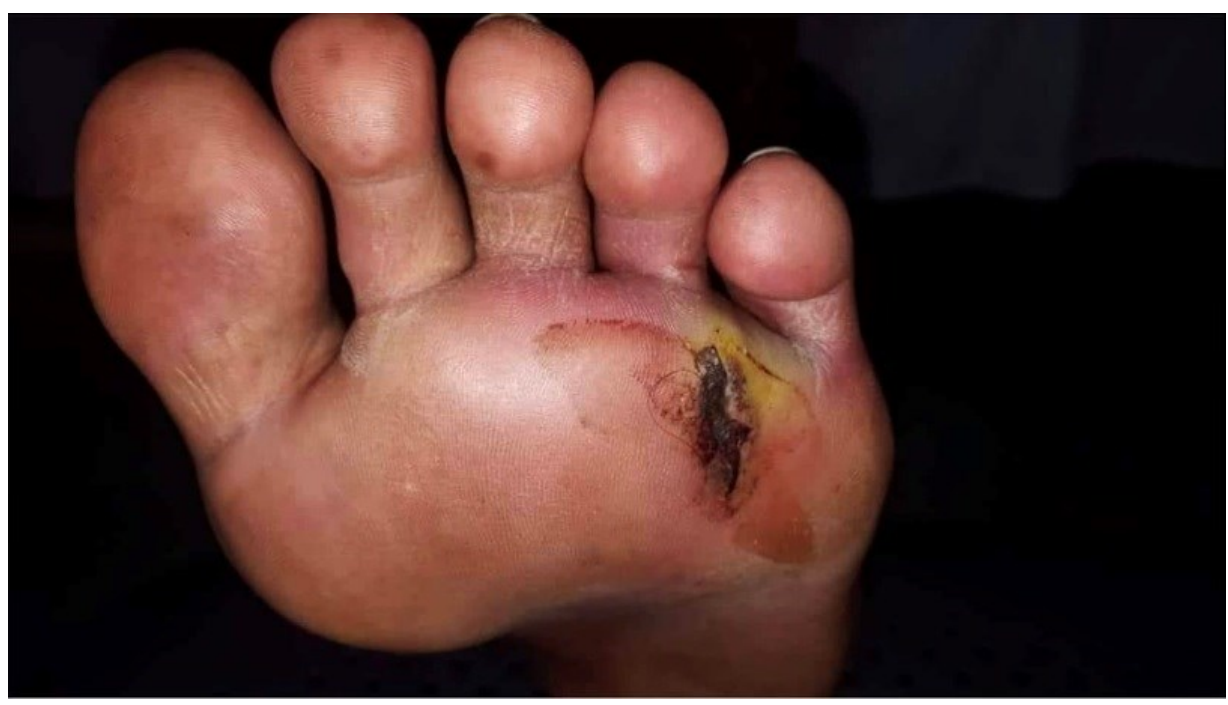


myocardial infarction in 2004 for which he underwent angioplasty with two stents in situ. In addition, the patient reported a 20 pack-year history and was unaware of his immunisation status.

Random blood sugar at this time was $350 \mathrm{mg} / \mathrm{dl}$ and oxygen saturation on room air at $98 \%$. On examination, the left leg was swollen with pitting oedema at the level of the ankle and a $4 \mathrm{~cm}$ wound with necrotic edges on the plantar surface of the left foot. The dorsalis pedis pulses were decreased bilaterally with sensory examination confirming peripheral neuropathy in a stocking distribution. Laboratory investigations showed a leucocytosis of $12.3 \times 10^{9} / \mathrm{L}$ with haemoglobin of $15.24 \mathrm{~g} /$ dL. Creatinine was $1.55 \mathrm{mg} / \mathrm{dL}$ with BUN and electrolytes within normal ranges. CRP was elevated at $23.8 \mathrm{mg} / \mathrm{L}$ with a Hba1c of $8.9 \%$ and normal CPK. An electrocardiogram showed an ejection fraction of $41 \%$ with left ventricular hypertrophy while $x$-rays of the left foot had no significant changes for osteomyelitis. A decision was made to perform femoral arteriogram of the lower limbs bilaterally as a result of the decreased dorsalis pedis pulses.

The patient was admitted to the medicine and general surgery service with a diagnosis of left diabetic foot gangrene secondary to peripheral vascular disease. The patient was started on meropenem and metronidazole. Additionally, 6000 units of tetanus immune globulin was administered intramuscularly. He underwent a femoralfemoral bypass and extensive debridement of the left foot wound under general anaesthesia. Postoperatively, the patient developed acute hypoxic respiratory failure secondary to decompensated heart failure. The patient complained of dysphagia and lockjaw 24 hours after admission. Examination revealed risus sardonicus and a reflex spasm on touching the oropharynx using a spatula which indicated a positive spatula test. A diagnosis of tetanus was made. Within 48 hours of admission, the patient developed facial spasms and worsening respiratory distress. He was intubated and started on midazolam and cisatracurium infusion along with further debridement and amputation of the first two metatarsals of the left foot. The patient developed autonomic dysreflexia 72 hours following admission and propranolol was given via NGT. Laboratory investigations revealed worsening acute kidney injury with creatinine of $2.79 \mathrm{mg} /$ dL. Supportive management was continued with gentle IV hydration, antibiotics and sedatives. Despite this, the patient died 96 hours after admission.

\section{DISCUSSION}

Tetanus is a life-threatening infectious disease caused by the bacterium Clostridium tetani. Tetanus is widespread in areas where there is poor immunisation coverage and in elderly patients as they fail to stay up-to-date with their vaccination schedules or due to declining levels of immunity. Tetanus is mainly found in soil, dust and animal excretions and is transmitted through the inoculation of spores in wounds, burns, ulcers, gangrene and abrasions of the skin. ${ }^{1}$ Spores release the exotoxin, tetanospasmin, which blocks the release of inhibitory neurotransmitters causing painful muscle contractions. ${ }^{2}$ The diagnosis of tetanus is made clinically based on presenting symptoms and risk factors and is supported by the lack of or outdated vaccinations, the absence of tetanus toxin antibodies and the culture of $C$. tetani from the wound site.

There has been a significant decrease in tetanus cases globally with the use of the tetanus toxoid vaccine which is both effective and inexpensive. However, tetanus is still common in low to middle income countries due to the lack of proper immunisation programs with estimated deaths in 2002 ranging between 213,000 to 293,000 persons (inclusive of 180,000 deaths from neonatal tetanus). ${ }^{3}$ In developed countries, tetanus is usually most prevalent in the elderly and intravenous drug users due to reduced immunisation levels and the use of contaminated needles or drugs respectively. According to the Centres for Disease Control and Prevention (CDC), a total of 33 tetanus cases and two deaths occurred in America in 2017 (the last year for which data is available), which is a significant decrease when compared to the 500 to 600 cases of tetanus that occurred in the 1940s and 1950s. ${ }^{4}$

Clinical tetanus is divided into three types: generalised tetanus, localised tetanus and cephalic tetanus. Generalised tetanus is the most common type and is characterised by trismus, risus sardonicus, opisthotonos, back and neck stiffness, dysphagia and restlessness. Patients may also develop autonomic instability which can lead to hemodynamic instability and cardiac arrest. Localised tetanus involves painful muscle spasms of muscle groups that are close to the wound site. Cephalic 
tetanus, the rarest form, results in cranial nerve palsies with flaccid paralysis as a result of contaminated wounds of the head or face. ${ }^{1}$ Both our patients in the present case series had symptoms of generalised tetanus.

Individuals that are at an increased risk for infection with tetanus include those who are unvaccinated and have contaminated puncture wounds, compound fractures, burns, ulcers or gangrene, the elderly, diabetics and intravenous drug users. ${ }^{1,5}$ The patient in Case 1 had multiple factors putting him at risk for tetanus infection including a past medical history of diabetes and an unvaccinated status alongside ongoing gangrene. Similarly, the patient in Case 2 had diabetes mellitus and an unknown immunisation status together with a contaminated wound.

The diagnosis of tetanus is entirely clinical and involves recognising the presenting signs of hypertonia associated with painful muscle contractions or spasms. Laboratory and imaging studies can be used to rule out differential diagnosis of hypertonia and muscle spasms but are not helpful in the diagnosis of tetanus. Differential diagnosis of tetanus may include strychnine poisoning, dental and local infections, malignant hyperthermia, dystonic drug reactions or seizure disorders. ${ }^{1}$ In many instances, the diagnosis of tetanus is not entirely straightforward due to ambiguous clinical features which may include fevers, diaphoresis, dysphagia and neck stiffness. This makes the diagnosis of tetanus challenging. Furthermore, as tetanus becomes an increasingly rare diagnosis, medical providers do not have the experience needed to diagnose and manage the condition effectively. Studies in England, the United States of America and Europe have shown that physicians have misdiagnosed patients with other conditions who are partially or incompletely immunised while not particularly paying attention to their immunisation status. ${ }^{6-8}$ This leads to a delay in treatment and even in some cases leads to the inability to provide simple prophylaxis.

Prompt treatment is recommended once the diagnosis of tetanus is made to prevent progression which may lead to respiratory failure or autonomic dysfunction resulting in cardiac arrest or hemodynamic instability. ${ }^{9}$ Initial treatment includes the administration of tetanus immunoglobulin which can neutralise any unbound circulating toxin, tetanus toxoid or antibiotics with anaerobic coverage such as metronidazole and aggressive wound debridement. ${ }^{10}$ Symptomatic treatment includes the use of benzodiazepines for muscle spasms and the use of a dark and quiet environment to prevent reflex spasms. ${ }^{1}$ More severe cases may warrant admission to the ICU with sedation and mechanical ventilation. Endotracheal intubation often poses difficulty as it can trigger muscle spasms and as such a tracheostomy is preferred especially in those patients who require ventilation for more than 10 days. Proper management of respiratory failure and autonomic dysfunction, which can be worsened by intensive care interventions, is important to ensure survival.

The prognosis of tetanus depends on the time from first symptom to onset of the first spasm. A shorter time to manifestation of symptoms along with onset of autonomic dysfunction is associated with a poorer prognosis. ${ }^{9}$ Those patients who recover have a prolonged recovery period with hypotonia and symptoms of autonomic dysfunction that lasts for years.

\section{CONCLUSION}

Even though tetanus is an increasingly rare diagnosis, it is important to be aware of the signs and symptoms so prompt treatment can be initiated. It is important that an immunisation history is taken from high risk populations which include the elderly, diabetics and intravenous drug users and those who present for wound care so that the possibility of misdiagnosis is reduced. This simple measure can ensure that this life-threatening disease can be prevented in high-risk populations.

Ethical Approval statement: Not applicable

Conflict of Interest statement: None

Informed Consent statement: Informed consent was obtained from all concerned parties

Funding statements: No sources of funding to declare Author Contributions: All listed authors contributed to this case series 


\section{REFERENCES}

1. Bleck TP. Tetanus: pathophysiology, management, and prophylaxis. Dis Mon. 1991;37(9):545-603.

doi:10.1016/0011-5029(91)90008-y

2. González-Forero D, Morcuende S, Alvarez FJ, de la Cruz RR, Pastor AM. Transynaptic effects of tetanus neurotoxin in the oculomotor system. Brain. 2005;128(Pt 9):2175-2188. doi:10.1093/brain/awh580

3. Roper $\mathrm{MH}$, Vandelaer JH, Gasse FL. Maternal and neonatal tetanus. Lancet (London, England). 2007;370 (9603):1947-1959. doi:10.1016/S0140-6736(07)61261-6

4. Centers for Disease Control and Prevention. Tetanus Surveillance, 2019. https://www.cdc.gov/tetanus/ surveillance.html\#figure (Accessed 10/06/2020)

5. Centers for Disease Control and Prevention. Tetanus, 2020. https://www.cdc.gov/tetanus/clinicians.html (Accessed 10/06/2020)

6. Savage EJ, Nash S, McGuinness A, Crowcroft NS. Audit of tetanus prevention knowledge and practices in accident and emergency departments in England. Emerg Med J. 2007;24(6):417-421. doi:10.1136/

emj.2007.047399

7. Tetanus surveillance --- United States, 2001-2008. MMWR Morb Mortal Wkly Rep. 2011;60(12):365-369.

8. Abbate R, Di Giuseppe G, Marinelli P, Angelillo IF. Appropriate tetanus prophylaxis practices in patients attending Emergency Departments in Italy. Vaccine. 2008;26(29-30):3634-3639. doi:10.1016/

j.vaccine.2008.04.072

9. Amare A, Melkamu Y, Mekonnen D. Tetanus in adults: clinical presentation, treatment and predictors of mortality in a tertiary hospital in Ethiopia. $J$ Neurol Sci. 2012;317(1-2):62-65. doi:10.1016/j.jns.2012.02.028

10. Campbell JI, Lam TMY, Huynh TL, et al. Microbiologic characterization and antimicrobial susceptibility of Clostridium tetani isolated from wounds of patients with clinically diagnosed tetanus. Am J Trop Med Hyg. 2009;80 (5):827-831. 